\title{
Congratulations on the launch of the Robotic Gynecologi- cal Surgery journal!
}

\author{
Hung-Cheng Lai ${ }^{1,2}$ \\ ${ }^{1}$ Department of Obstetrics and Gynecology, Shuang Ho Hospital, Taipei Medical University, New Taipei City; ${ }^{2}$ Department of Obstetrics and \\ Gynecology, Taipei Medical University, Taipei, Taiwan
}

With the advancement of robotic surgeries in various disciplines, robotic gynecological surgery is one of the most mature specialties. It is superseding urology in case numbers in many countries. Despite the pioneering role of gynecology in the history of robotic surgery development and the establishment of various societies for robotic gynecological surgeons worldwide, there is still no focus journal on this subspecialty. With the broader adoption of this evolving technology in gynecology in different countries and at a different pace, a journal dedicated to this field for education, experience sharing, clinical studies, and trials is needed. My sincere congratulations go to Dr. Cho and his colleagues from the Society of Korean Robotic Gynecology Surgery (SKRGS), who made a dream come true. The RGS journal's launch will be a milestone, which will bring people dedicated to robotic gynecological surgery around the world and shape the future of gynecological surgery together. The Asian Society for Gynecologic Robotic Surgery (ASGRS) will be an alliance and a strong backup for RGS.

With Warm Regards,

Hung-Cheng Lai, MD, PhD

President, Asian Society for Gynecologic Robotic Surgery

- Received: August 30, 2020 • Accepted: September 1, 2020

- Correspondening author: Hung-Cheng Lai

Department of Obstetrics \& Gynecology, Shuang Ho Hospital, Taipei Medical University, No.291, Zhongzheng Rd., Zhonghe District, New Taipei City 23561, Taiwan

E-mail:hclai30656@gmail.com

This is an Open Access article distributed under the terms of the Creative Commons Attribution Non-Commercial License (http://creativecommons.org/ licenses/by-nc/4.0) which permits unrestricted non-commercial use, distribution, and reproduction in any medium, provided the original work is properly cited. 